\title{
Une conférence de contrastes, de richesses et de menaces
}

«Sauvegarde et développement de la langue et de la littérature des petits peuples du Nord de l'okroug autonome khanty-mansi - Jugra, protection de l'héritage culturel - Fondements légaux et problèmes »

\section{Catherine Le Roux}

\section{(2) OpenEdition}

12 Journals

Édition électronique

URL : https://journals.openedition.org/efo/4342

DOI : $10.4000 /$ efo. 4342

ISSN : 2275-1947

Éditeur

INALCO

Édition imprimée

Date de publication : 1 janvier 2014

ISBN : 978-2-343-05394-3

ISSN : 0071-2051

\section{Référence électronique}

Catherine Le Roux, «Une conférence de contrastes, de richesses et de menaces », Études finnoougriennes [En ligne], 46 | 2014, mis en ligne le 15 octobre 2015, consulté le 08 juillet 2021. URL http://journals.openedition.org/efo/4342 ; DOI : https://doi.org/10.4000/efo.4342

Ce document a été généré automatiquement le 8 juillet 2021.

\section{(c) (7) \&}

Études finno-ougriennes est mis à disposition selon les termes de la Licence Creative Commons Attribution - Pas d'Utilisation Commerciale 4.0 International. 


\section{Une conférence de contrastes, de richesses et de menaces}

«Sauvegarde et développement de la langue et de la littérature des petits peuples du Nord de l'okroug autonome khanty-mansi - Jugra, protection de l'héritage culturel - Fondements légaux et problèmes $»^{1}$

\section{Catherine Le Roux}

Du 2 au 4 juillet 2013 s'est tenue à Kogalym (Sibérie Occidentale) une conférence internationale intitulée « Sauvegarde et développement de la langue et de la littérature des petits peuples du Nord de l'okroug autonome khanty-mansi - Jugra, protection de l'héritage folklorique - Fondements légaux et problèmes ".

2 La conférence était organisée dans le cadre du $20^{\mathrm{e}}$ anniversaire de la Duma (Assemblée de députés) de l'okroug Jugra² qui sera célébré le 6 avril 2014. Présidée par Eremej Ajpin, à la fois vice-président de la Duma et président de l'Assemblée des représentants des " petits " peuples autochtones du Nord, elle a réuni une cinquantaine de participants : des membres - élus et administratifs - de la Duma, des représentants de l'exécutif du district, des personnalités de la ville de Kogalym, trois représentants de la compagnie pétrolière Lukoil, des éleveurs de rennes (certains venus avec femme et enfants) ainsi que le président et le vice-président de l'Union des éleveurs de rennes, des membres d'associations de sauvegarde de la région, des représentants des peuples autochtones, des ethnologues, des enseignants, des juristes, des écrivains, des journalistes, etc. Des chercheurs et traducteurs de Finlande, France et Hongrie étaient également présents.

3 En introduction à la conférence proprement dite, les participants se sont vu proposer une rapide excursion dans la ville de Kogalym avec, notamment, une visite du centre culturel, à la fois musée et centre d'exposition. Kogalym est située dans l'okroug autonome khanty-mansis, sur la rivière Ingujagun, à $325 \mathrm{~km}$ au nord-est de HantyMansijsk. Fondée en 1975 pour accompagner le développement de l'extraction du pétrole et du gaz de la région, la ville compte aujourd'hui près de 60000 habitants $^{3}$, son économie repose sur la principale entreprise de la ville: Lukoil-Zapadnaja Sibir' ${ }^{4}$. Lukoil a d'ailleurs largement contribué au financement de la conférence. 
4 Assurée par la mairie, la visite de la ville et du musée/centre d'exposition s'est faite dans le droit fil de la tradition russe: professionnalisme de la guide, abondance de chiffres, souci de valoriser une ville malgré le handicap d'un court passé qui la prive d'atouts historiques ou culturels... Et, dans le même temps, rien de spécifique pour un auditoire venu participer à une conférence sur les peuples autochtones de la région. La visite du musée/centre d'exposition fut à cet égard un modèle du genre : l'essentiel de la visite fut consacré à la partie "moderne» du centre - exposition sur l'industrie pétrolière, espaces récréatifs pour les jeunes avec présentation d'installations et animations diverses - pour n'aborder qu'en toute fin le «musée » proprement dit, consacré aux peuples autochtones. Déception! Tant les objets exposés que les explications données sur les cartels ou par la guide relevaient d'une approche «folklorique » superficielle, et désinvolte car présentant des erreurs : on était bien loin du souci de rendre présents et de valoriser les peuples autochtones.

Le soir, un buffet/soirée poétique - célébrant notamment le $65^{\mathrm{e}}$ anniversaire

d'Eremej Ajpin - fut l'occasion d'entendre des poèmes et des chants : compositions russes d'habitants locaux ou chants khantys et mansis chantés par des autochtones. Contraste d'une prestation littéraire mêlant femmes russes dont la vie à Kogalym est étroitement liée à l'industrie pétrolière et poèmes et chants dits par des autochtones à la survie menacée.

5 Dès cette première soirée se profilaient les contrastes, l'ambivalence qui allaient traverser ces quatre jours: une conférence internationale sur la sauvegarde de la langue et de la littérature des petits peuples de l'okroug autonome khanty-mansi est organisée dans une ville qui n'a aucun lien avec ces peuples; elle bénéficie du soutien et du financement de la compagnie pétrolière Lukoil ainsi que de la ville, créée pour l'exploitation de ce pétrole, à la fois manne pour la Russie et menace pour les autochtones.

\section{La conférence}

6 La conférence elle-même s'est ouverte le 3 juillet sous la présidence d'Eremej Ajpin. Après la prière rituelle adressée, selon la tradition mansie, aux dieux de la terre de Jugra par la poétesse Svetlana Dinislamova pour le bon déroulement de la conférence, les personnalités locales officielles ont prononcé les introductions d'usage: Eremej Ajpin sur la langue et sur le rôle de la Duma, Igor Širmanov, conseiller du président de la Duma, sur la dimension législative de l'action de cette dernière; Ljudmila Alfërova, conseiller du premier gouverneur adjoint de Jugra, Gennadi Buhtin, a lu le message de bienvenue de ce dernier et annoncé la parution en 2014 de nouvelles revues pour enfants ${ }^{5}$, Konstantin Beljaev, représentant de Lukoil Sibérie occidentale, a présenté l'action de sa compagnie en faveur des peuples autochtones. Aucune de ces interventions ne donna matière à débat.

7 La parole fut ensuite donnée aux invités, étrangers et moscovites, qui présentèrent ce qui se fait chez eux dans le domaine de la culture et des langues du Nord. Marjo Mäenpää, conseiller au ministère de l'Éducation et de la Culture de Finlande, présenta les actions de son ministère dans les domaines de la culture same et de la coopération culturelle avec la région de Hanty-Mansijsk; Ferenc Kereszti retraça comment, jeune ingénieur informaticien hongrois envoyé à Hanty-Mansijsk pour une mission sur l'exploitation pétrolière, il se vit recommander un certain nombre de contacts autochtones par des ethnologues hongrois: heureux de pouvoir ainsi 
contourner les contraintes de l'époque, ceux-ci lui permirent de faire la connaissance d'Eremej Ajpin et, grâce à lui, de nombreux autres autochtones ; Julianna Kissné Rusvai évoqua les traductions hongroises des auteurs khantys faites par Eva Schmidt; pour la France, Eva Toulouze présenta l'activité de l'ADEFO (Association pour le Développement des Études Finno-Ougriennes) et Dominique Samson Normand de Chambourg souligna que l'INALCO ( Institut National des Langues et Civilisations Orientales) collabore de façon active depuis dix ans avec les peuples autochtones et dispense des cours d'initiation aux langues de Sibérie, notamment un cours de nenetse ; Vjačeslav Ogryzko, rédacteur en chef de La Russie littéraire, souleva le problème des ressources insuffisantes pour financer des rééditions, qui touche également les écrivains du Grand Nord.

8 Le manque de moyens sera un thème récurrent de la conférence, avec des désaccords sur les moyens de trouver des ressources nouvelles : miser - par conviction mais sans grand espoir - sur des ressources budgétaires en sollicitant les autorités locales ou fédérales, ou bien prendre l'argent là où il se trouve, c'est à dire auprès de Lukoil ?

9 Ce fut ensuite aux autochtones ou aux participants travaillant en lien direct avec eux d'intervenir. Quelques interventions ont été particulièrement notées :

Juri Vella présenta son Dictionnaire toponymique de la rivière Agan et de ses affluents dont le troisième tome venait de sortir. Pour lui, la toponymie est riche d'informations sur l'histoire, sur ce qu'on peut faire, sur ce qui est interdit... Toute personne qui veut pénétrer dans la région, s'informer sur elle, doit respecter ces messages, les rites qu'ils évoquent, au risque d'être punie, comme ce fut le cas de l'ethnologue russe Raisa Mitusova, dont Vella retraça l'histoire ${ }^{6}$. Igor Širmanov, titulaire de la chaire de jurisprudence de l'université de Nižnevartovsk, traita du droit coutumier chez les peuples du Nord.

11 La matinée du deuxième jour fut particulièrement animée : les oratrices parlèrent avec passion et résistèrent avec détermination à Eremej Ajpin qui tentait à la fois d'abréger leur exposé (pour des questions de temps) et d'occulter certaines questions, considérant qu'elles n'avaient pas à être abordées dans le cadre de la conférence (malgré l'avis des oratrices ainsi que d'une partie du public), car facilement résolubles avec la participation financière de LUKOIL. Larisa Mineeva, enseignante de Langepas, souligna avec vigueur et conviction le manque de supports méthodologiques pour enseigner les cultures et langues locales. Depuis 1998 - année où, géographe de formation, elle fut chargée d'enseigner l'histoire et la culture de la région de l'ob' -, Larisa Mineeva, elle-même de nationalité russe, poursuit avec passion la mission de faire découvrir aux enfants de cette région les richesses de la culture des Ougriens de l'ob', de faire naître chez les autochtones la fierté d'appartenir aux peuples khanty et mansi, et chez les enfants russes le respect pour cette culture et cette civilisation. En l'absence de supports pédagogiques, elle s'est attachée à en créer en utilisant et en développant toutes les sources possibles d'information: articles scientifiques, écrits d'auteurs et de poètes khantys et mansis (notamment Ajpin et Šestalov ${ }^{7}$ ), contacts avec des personnalités. Elle a également créé en 1999, avec d'autres, l'association « Sons de la terre ougrienne ", pour approfondir et faire connaître la culture des peuples du Nord : formations, expositions, visites de villages autochtones, rencontres, manuels et ouvrages divers ${ }^{8}$, travaux de recherche primés dans différents concours, centre ethnographique présentant les réalisations des enfants sur leur région, initiation aux métiers artisanaux avec le concours d'artisans du village Agan, films, etc. Convaincue 
qu'un enseignement sur la civilisation des Ougriens de l'ob' est une richesse pour tous et pas seulement pour les écoliers autochtones (très peu nombreux), et qu'il s'inscrit très bien dans le Programme éducatif de la Fédération de Russie jusqu'en 2025 (qui assigne à l'enseignement la mission de surmonter la crise actuelle des valeurs), Larisa Mineeva lance un appel aux participants pour l'aider à trouver les moyens nécessaires à la poursuite de l'édition de manuels pour les écoliers au-delà de la $5^{\mathrm{e}}$ classe.

Maja Makarova, de l' "Institut de développement de la formation» du district de Hanty-Mansijsk, a présenté le "Centre de formation ethnoculturel pour enfants "Lylyng Sojum », qui élabore et édite des supports méthodologiques d'enseignement, organise des séminaires pour les enseignants sur l'intégration de la composante ethnoculturelle dans les programmes de formation, etc. Maja Makarova a, elle aussi, souligné le manque de crédits pour ces programmes et supports de formation. Créé à Hanty-Mansijsk en 2003 pour transmettre aux jeunes générations urbanisées les traditions et valeurs des Ougriens de l'Ob', Lylyng Sojum est, depuis 2007, Centre municipal de formation extrascolaire pour enfants : musique instrumentale, chants et danses populaires, théâtre, peinture et arts décoratifs appliqués, mais également enseignement des langues autochtones à travers des activités ludiques et artistiques. Devenu partie intégrante du système éducatif de la ville de Hanty-Mansijsk, il a dû développer méthodes et supports spécifiques et recruter des spécialistes connaissant les us et coutumes des peuples autochtones. Depuis 2008, un programme "Développement de l'enfant à partir des traditions populaires des Ougriens de l'ob' " concerne non seulement les enfants autochtones mais aussi les non autochtones des jardins d'enfants et écoles de la ville. Le centre a également sensibilisé l'opinion à la sauvegarde des traditions populaires et contribué à accroître la considération pour les autochtones urbanisés. Un centre de ressources élabore et met à disposition des supports pédagogiques pour les différentes activités, une base de données des métiers artisanaux, des matériaux ethnographiques et folkloriques des langues. Tout cela a permis d'ouvrir un Musée qui organise expositions et manifestations. L'équipe souhaite aujourd'hui élargir l'activité du Centre à la sphère scolaire à proprement parler et a déjà mis au point un programme éducatif couvrant toutes les classes.

13 Agrafena Sopočina (Ljantor), directrice de l'Institut ougrien de l'ob' de recherche appliquée (filiale de Surgut), aborda la graphie du dialecte khanty de Surgut et les problèmes pour l'enseigner. Cet exposé très technique ne peut être résumé mais l'oratrice a su intéresser les non spécialistes en rappelant le contexte linguistique: chaque groupe de dialectes khantys constitue une langue distincte avec sa phonétique, sa grammaire, sa sémantique. Celui de Surgut est largement utilisé, non seulement dans la vie quotidienne mais également dans les écoles et pensionnats, les organismes officiels, l'administration des villes et des villages, les tribunaux, les musées, bibliothèques, etc. (plus de $90 \%$ des Khantys de Surgut parlent leur dialecte). Depuis 2010 , l'introduction de «PT Serif» dans les polices des ordinateurs a beaucoup facilité la translittération des textes de l'oralité, des manuels scolaires et des œuvres littéraires, mais les obstacles à l'enseignement des dialectes orientaux dans les écoles restent inchangés: spécialisation trop étroite des départements et de ceux qui y travaillent, absence d'outils techniques modernes, absence de budget pour former des spécialistes des dialectes orientaux khantys, absence de programmation pour la recherche et l'élaboration de supports pédagogiques (manuels divers, dictionnaires, etc.). Déplorant l'absence de spécialistes de khanty oriental en Russie et le désengagement de l'université de Jugra, Agrafena Sopočina a précisé que la Hongrie 
dispose, elle, de quatorze spécialistes du domaine et qu'une coopération devrait s'instaurer.

14 Ces dernières interventions, très vives dans le fond et dans la forme, ont vivement intéressé les participants. Et on regrette que le président de séance n'ait pas souhaité engager la discussion sur les moyens de soutenir l'activité décrite, dont on a vu qu'elle reposait beaucoup sur des énergies individuelles et se heurtait à la fois à l'absence de moyens et à l'absence de politique officielle de soutien. Sur le point précis du manque de ressources financières, le correspondant de Lukoil a rappelé que sa compagnie était prête à affecter des crédits à la formation.

Alexandr Puryšev, conseiller d'Eremej Aipin à la Duma, intervint ensuite sur «Les territoires d'exploitation traditionnelle de la nature, périmètres de sauvegarde de la langue, de l'héritage folklorique et des sanctuaires des peuples de Jugra - Aspects juridiques ». Après s'être s'élevé vigoureusement - en réaction au débat précédent contre l'idée de chercher auprès de Lukoil des financements qui devraient être affectés par les pouvoirs locaux, régionaux et fédéraux, Alexandr Puryšev a traité de la situation des "territoires naturels spécialement protégés ${ }^{9}$ et des "territoires d'exploitation traditionnelle de la nature $»^{10}$ dans l'okroug autonome khanty-mansi ${ }^{11}$; ces territoires où s'exercent les activités traditionnelles - élevage de rennes, pêche, chasse, cueillette - voient leurs limites menacées par les progrès de l'exploitation industrielle. Une menace pèse, en outre, sur les sanctuaires (lieux sacrés où se pratiquent des rites religieux) des petits peuples autochtones : d'une part, c'est le gouvernement du district qui a tout pouvoir ${ }^{12}$ pour créer les conditions juridiques, organisationnelles, matérielles, financières et autres de leur entretien et de leur sauvegarde ; d'autre part, ils doivent être déclarés héritage culturel ou inscrits comme héritage culturel du district à l'inventaire culturel ou historique général des peuples de la Fédération de Russie.

Compte tenu du retard pris lors de la première journée, la fin de la conférence en moitié de deuxième journée fut précipitée: certains orateurs prévus ne purent intervenir, le débat final fut remplacé par la remise d'un document exposant les « recommandations de la conférence », dont certaines adressées à la Duma et les autres à l'exécutif du district.

17 Ce fut ensuite le départ des participants vers les campements: les participants étrangers étaient invités au campement appartenant à la famille d'Eremej Ajpin, les autres se rendaient au campement touristique dit Karamkin.

\section{Les visites de campements}

\section{Le campement de la famille d'Eremej Ajpin}

18 De Kogalym, nous avons été emmenés au campement par deux hélicoptères de la compagnie Lukoil (Eremej Ajpin, le rédacteur en chef de la revue Russie littéraire et six des participants étrangers à la conférence).

Le campement est situé près d'un affluent de l'Agan et compte plusieurs bâtiments, tous en rondins : l'un, assez grand, construit récemment, sert de lieu de couchage, un autre pour les repas, un pour les bains, deux labaz (où on préserve nourriture et équipements saisonniers), un abri pour les rennes, un autre, plus petit et à l'écart, pour 
les toilettes. Tous sont disposés dans une clairière, deux autres se dressent un peu plus loin, au milieu des arbres.

Nous sommes accueillis à la descente de l'hélicoptère par la femme d'Ajpin (russe, médecin en retraite), par la sœur aînée du député, Elizaveta, le fils de celle-ci, Serioža, et son mari Pavel Ajvaseda. Elizaveta et son mari vivent à Var'ëgan mais sont venus pour nous accueillir. Nous accueillent également les moustiques, costauds et nombreux: ils ne nous lâcheront que lorsque nous serons dans les intérieurs, aux fenêtres et portes fermant hermétiquement.

21 Arrivés en début d'après-midi, nous resterons dans le campement jusqu'au milieu de la matinée suivante : visite du campement, promenade dans la taïga alentour, observation des rennes qui alternent repos allongé près du «dymokur "- poêle alimenté en permanence d'herbe sèche et dont la fumée doit éloigner les moustiques -, déplacement tranquille dans l'enclos et course soudaine vers la taïga : l'enclos, marqué par des barrières basses, n'est pas fermé, les rennes paraissent libres de leurs mouvements, de jour comme de nuit.

C'est Serioža, le neveu d'Ajpin, qui tient le campement sans y vivre en permanence. À la fois très occupé et de tempérament réservé, il se tient à l'écart et parle très peu ${ }^{13}$. Au soleil couchant, il nous emmènera faire un grand tour en barque à moteur (c'est Lukoil qui fournit le carburant, dans le cadre des dédommagements) sur la rivière, un affluent de l'Agan, puis sur l'Agan même. Les moustiques, bien sûr, nous accompagnent, mais la nature est splendide, se reflète dans l'eau apparemment très propre, avec ici et là des constructions en bois pour les pêcheurs.

23 Les échanges auront lieu essentiellement avec Pavel Ajvaseda, et, dans une moindre mesure, avec Elizaveta, très préoccupée de nous accueillir le mieux possible et s'affairant sans cesse pour aller chercher de l'eau, la faire chauffer pour le thé et préparer d'incessantes libations. La rusticité des installations fait que tout ceci prend du temps, même si nous avons essayé d'aider le plus possible ${ }^{14}$.

Pavel a plus de 80 ans, il attribue sa vitalité à sa sobriété et dit être l'homme le plus âgé de Var'ëgan, tous ses congénères ayant été tués - directement ou indirectement - par l'alcool : accidents, suicides, etc. Très disert, heureux d'avoir des auditeurs, il raconte volontiers sa vie, répond à nos questions - sur les ours, sur les rennes, mais, comme le veut la tradition, ne donne pas l'importance du troupeau si ce n'est de manière détournée par des comparaisons, des anecdotes, des devinettes, etc.

Les devinettes, nous en aurons à nouveau, à la veillée, jusqu'à une heure assez avancée : nous sommes tous allongés sur les bat-flanc de la maisonnette-chambre; à une extrémité, des couvertures accrochées au plafond forment une alcôve pour Elizaveta et son mari. Celui-ci prend alors un fauteuil, s'assied devant les bat-flanc et entame une longue série de devinettes khantyes. Celui d'entre nous qui trouve gagne des objets artisanaux : mini-tête de renne faite d'un bec de canard et de petits os, pattes articulées de poule arctique...

26 La matinée du lendemain débute par un petit-déjeuner nourrissant (pot-au-feu de renne avec des nouilles), préparé par une femme d'un campement voisin. Nous attendons ensuite l'hélicoptère qui nous emmènera au campement Karamkin : nous nous promenons dans la clairière, observant les allées et venues des hommes et des rennes. Au cours de ces 24 heures, nous resterons observateurs discrets : seuls auront des contacts plus approfondis Dominique Samson - familier des lieux pour y avoir déjà 
séjourné et participé à la construction d'un des bâtiments, qui parlera longuement avec Elizaveta - et Ogryzko, lui aussi déjà venu, qui interviewera Pavel.

\section{Le campement Karamkin}

27 Ce campement, appelé aussi « ethnocampement Karamkin »"15 est situé au nord-ouest de Nižnevartovsk, au bord d'un affluent de l'Agan. Il est tenu par Ljubov Kazandži ${ }^{16}$, originaire du village d'Agan, qui a décidé, en mars 2011, de l'ouvrir aux touristes. Elle a, à ce titre, bénéficié d'une subvention de 2 millions de roubles (45000 euros environ) au titre du «meilleur projet touristique». Son campement est devenu l'une des curiosités de la région. Ljubov Kazandži nous accueille, entourée de femmes khantyes et nenetses des forêts, toutes habillées de vêtements locaux, colorés, brodés. On nous fait visiter : le contraste avec le campement d'Ajpin est saisissant.

Tout d'abord, ô surprise, pas de moustiques : nous apprîmes que ceux-ci avaient été «éloignés » (éradiqués ?) pour deux jours, le temps de la visite. Les bâtiments, ensuite : par rapport au campement Ajpin où les constructions paraissaient avoir été là de tout temps, ici elles ont un air pimpant, rénovées ou copies de constructions traditionnelles - la cuisine, le bâtiment où l'on dort, le labaz, un «tchoum » flambant neuf, fort beau au demeurant, et, à l'arrière, les rennes. Eux sont bien authentiques, paraissent accablés par la chaleur (ou assoupis par le produit anti-moustiques?) Devant le tchoum, une petite exposition d'objets artisanaux présentés à la vente. Des vêtements traditionnels sont mis à la disposition des visiteurs pour les endosser et se faire photographier. À l'intérieur, on nous fait asseoir ; une femme, accompagnée de deux garçonnets - venus au campement pour l'occasion - nous présente une cithare (la cithare khantye, narsjuh), nous en montre le maniement, nous fait essayer: nous grattons quelques notes et lui demandons d'en jouer elle même ou de chanter en s'accompagnant. Nous n'obtiendrons pas grand-chose. Timidité? La femme est pourtant très accueillante. Connaissance limitée des chants traditionnels?

À l'extérieur, on nous propose deux jeux de bâton: pour le premier, deux personnes sont debout, face à face, les pieds croisés et tiennent, juste au-dessus du genou, un grand bâton type manche à balai, qu'elles doivent tirer avec leurs cuisses sans se servir des mains; pour le deuxième, deux personnes sont assises face à face, les jambes tendues, tiennent un bâton dans leurs mains et tirent chacune de son côté afin de faire perdre l'équilibre à l'adversaire.

Nous sommes ensuite invités à une collation: brochet fraîchement pêché dans la rivière, servi grillé, délicieux, mais également du «stroganina » (copeaux congelés) de brochet, de l'infusion de canneberge et le traditionnel thé. Les quelques Russes présents proposent de la vodka, au grand dam de ceux - locaux ou étrangers - qui connaissent bien les ravages de l'alcool chez les autochtones.

Impression générale mitigée : dans cette ouverture du campement aux visiteurs, au tourisme, conçue pour contribuer à la survie du campement et, à travers lui, d'une part de la culture khantye, le goût de l'exotisme l'emporte à l'évidence - certaines des nouvelles constructions ne respectent pas les règles traditionnelles, tel le labaz situé en milieu de campement et derrière lequel il faut passer pour retrouver les rennes alors que la tradition l'interdit; les « locaux » présents ne semblent pas avoir d'autre activité que l'accueil des visiteurs et les visiteurs eux-mêmes ne se voient pas proposer d'autre activité que les jeux de bâton; les chants ou contes khantys que l'on veut nous montrer 
ne sont connus qu'à moitié. Peut-être est-ce défaut de jeunesse : on pourrait souhaiter que l'ensemble respecte et restitue davantage le mode de vie des éleveurs de rennes, mais comment faire quand on accueille 120 personnes à la fois ?

Le retour à Kogalym se fait, dans un véhicule utilitaire affrété par Lukoil, sur une route plus que cahotante, construite pour l'exploitation pétrolière.

\section{Conclusion : Une conférence de contrastes et de richesses dans un contexte bien menacé}

- Une conférence sur la préservation du patrimoine autochtone qui se tient dans une ville, Kogalym, sans lien aucun avec ce patrimoine : ville en pleine zone d'exploitation pétrolière, où la relation aux autochtones est dominée par la superficialité - jeunes filles en costume local et faux ours à l'aéroport, approximations dans la partie « ethnographique » du musée ;

- Une conférence qui réunit des participants très divers entre lesquels le dialogue s'instaure mal :

- des représentants de la ville et de l'okroug qui tiennent un discours formel ;

- des représentants de l'industrie pétrolière: ils ont contribué au financement de la conférence, ne se dérobent pas au dialogue, publient dans leur revue ${ }^{17}$ un compte-rendu détaillé... tout en lui donnant un intitulé à la fois réducteur et non sans ambiguïté, «ФольклоР в ЗАКонЕ ». Littéralement, ce titre signifie «le folklore dans la loi » : prime donc au folklore puisque seule la première des onze recommandations de la conférence concerne l'adoption d'une loi sur le folklore. Mais il pourrait aussi se traduire par «c'est le folklore qui fait la loi », car l'expression est calquée sur celle liée au monde criminel des années 30, «воры В ЗАКонЕ» (« les voleurs font la loi ») : une élite du milieu criminel, groupe très fermé et porté vers la conspiration, observe des codes extrêmement stricts tels que l'absence de relations avec des femmes et avec les services de l'État;

- des témoignages d'autochtones ou de chercheurs et pédagogues, passionnés et passionnants, mais le sentiment d'une opportunité d'échanges manquée: mauvaise gestion du temps? Priorité aux officiels, aux étrangers? Réticences du président de la conférence?

- Un patrimoine bien menacé :

- Par l'abandon du mode de vie auquel il était lié : sédentarisation, urbanisation, mixité des mariages. L'industrie pétrolière y joue, certes, une large part, mais fait-elle autre chose qu'accélérer une évolution inéluctable?

- Par sa diversité : pour ne prendre que l'exemple de la langue, peut-on parler de la langue khantye quand les enfants et l'enseignant parlent des dialectes qui non seulement diffèrent les uns des autres, mais diffèrent également des rares supports pédagogiques que quelques passionnés ont élaborés et transmettent?

- Par la difficulté de préserver ce qui reste: manque de moyens, sans doute, mais aussi danger d'une «folklorisation» non professionnelle comme dans l'« ethnocampement Karamkin ».

- Une ambivalence permanente et profonde à l'égard de l'industrie pétrolière. 


\section{NOTES}

1. En russe : ЗАКОНОДАТЕЛЬНОЕ ОБЕСПЕЧЕНИЕ И ВОПРОСЫ РАЗВИТИЯ ЯЗЫКА, ЛИТЕРАТУРЫ И СОХРАНЕНИЯ ФОЛЬКЛОРНОГО НАСЛЕДИЯ КОРЕННЫХ МАЛОЧИСЛЕННЫХ НАРОДОВ СЕВЕРА ХАНТЫ-МАНСИЙСКОГО АВТОНОМНОГО ОКРУГА - ЮГРЫ.

2. L'okroug autonome khanty-mansi - Jugra est un sujet fédéral de Russie (dans l'oblast de Tjumen'). Il a une superficie de $523100 \mathrm{~km}^{2}$, est peu densément peuplé, avec seulement 1432817 habitants (2002). La capitale est Hanty-Mansijsk (53953 habitants), les plus grosses villes sont Surgut (285 027 hab.), Nižnevartovsk (239044 hab.) et Neftejugansk (107 830 habitants). Les langues locales, le khanty et le mansi, sont coofficielles dans la région avec le russe. Le district produit plus de $25 \%$ du PNB, extrait $6 \%$ du pétrole mondial et plus de $56 \%$ du pétrole russe.

3. 58913 en 2008.

4. En russe : ООо "ЛУКОЙЛ-ЗАПАДНАЯ СИБИРЬ").

5. "ШовРИЕ" en langue khantye ("ЗАЙчИк" en russe, «le lièvre» en français), "ВИТСАм" en langue mansie ("КАПЕЛЬКА" en russe « la gouttelette » en français), ainsi qu'un journal en dialecte de Surgut.

6. Raisa ne se souciait pas - si tant est qu'elle les connaissait - des règles non écrites des Khantys et des Nenetses des forêts. Elle entreprit ainsi une expédition dans la région en ne respectant pas le rite qui veut qu'on suive la course du soleil, et ne tint nul compte du tonnerre qui gronda en signe d'avertissement lorsqu'elle arriva à l'Agan. En quête d'objets à rapporter pour le musée russe, elle s'empara d'une idole. Un troisième coup de tonnerre retentit. Personne ne lui envoya d'autres signes. En 1937 elle fut arrêtée et fusillée. C'est ainsi, sans commentaire, que Juri Vella termina son intervention.

7. Juvan Šestalov (1937-2011), écrivain et poète mansi.

8. «La culture spirituelle du peuple khanty», "Géographie du district autonome de Hanty-Mansijsk» «Histoire du district autonome de Hanty-Mansijsk», «Nous, enfants de la nature».

9. En rusSe : ОСОБО ОХРАНЯЕМЫЕ ПРИРОДНЫЕ ТЕРРИТОРИИ - ООПТ.

10. En russe : ТЕРРИТОРИИ ТРАДИЦИОННОГО ПРИРОДОПОЛЬЗОВАНИЯ ТТП.

11. L'okroug autonome khanty-mansi compte 475 ТТП.

12. Article 6 de la loi № 92-03 du 8 novembre 2005.

13. L'article publié par Vjačeslav Ogryzko dans La Russie littéraire (Literaturnaja Rossija) apporte, entre autres, les précisions suivantes : «Serguei tient le campement depuis depuis 1997, après avoir conduit un bulldozer pour l'industrie pétrolière ; il est fier d'avoir montré qu'un éleveur peut tenir un campement sans parler le khanty. Le khanty, il ne le parle pas mais le comprend et souhaiterait que ses enfants l'apprennent, mais les manuels sont en dialecte de Surgut. Ne le comprenant pas, les enfants préfèrent le dialecte de l'Agan. Quant à l'institutrice, elle est d'Ugut, reprend les enfants pour qu'ils parlent comme elle, et transpose tout en dialecte d'Ugut ».

14. Dans son article, V. Ogryzko rapporte de nombreux détails sur la famille d'Ajpin qu'il tient d'Elizaveta: sa mère avait eu trois enfants avant de perdre son mari - le demi-frère Galaktion, dont elle a longtemps ignoré que c'était un demi-frère - et 
d'épouser le père d'Elisaveta. Ensemble, ils ont quatre enfants et mènent une vie traditionnelle - de la maison de mousse dans la forêt l'hiver au tchoum quand la neige commence à fondre puis l'été au bord de la rivière. Jusqu'à l'automne 1956, où la mère tombe mystérieusement malade et meurt rapidement (victime d'un mauvais sort, jeté par vengeance par un voisin ?) Elizaveta a 11 ans, Eremej 8, et derrière, Daša et Ol'ja. Une femme médecin croit ensuite bien faire en envoyant les trois petites filles à l'internat, laissant le père effondré, seul avec Eremej.

15. En russe : этНостоЙБИще «КАРАМКИнсКОЕ»

16. Cf. interview de Ljubov Kazandži

http://ugra-news.ru/print/31587

17. Neftjanik (« Le pétrolier »), $\mathrm{n}^{\circ} 27,12$ juillet 2013.

\section{INDEX}

disciplines khanty, mansi, russe, nénetse

nomsmotscles Hongrois, Khantys, Mansis, Russes

Mots-clés : édition, éducation, ethno-tourisme, industrie pétrolière, littérature, poésie Index géographique : Agan (rivière), Finlande, France, Ob' (rivière), Hongrie, Ingujagun (rivière), Kogalym, Langepas, Liantor, Nižnevartovsk, Neftejugansk, Khanty-Mansï̈sk (Ougrie) (district autonome), Fédération de Russie, Sibérie occidentale, Surgut, Tioumen, Ugut, Var'ëgan Keywords : publications, Education, ethno-tourism literature, oil industry, poetry, minority studies, twenty-first century (beginning), Khanty, Mansi, Russian, Nenets, Hungarians, Russians Thèmes : minority studies

Index chronologique : XXIe siècle, XXIe siècle (début) 